\title{
Single-Crystal MgO Hollow Nanospheres Formed in RF Impulse Discharge Plasmas
}

\author{
Satoru Iizuka and Takumasa Muraoka \\ Department of Electrical Engineering, Graduate School of Engineering, Tohoku University, \\ Aza-Aoba 6-6-05, Aramaki, Aoba, Sendai 980-8579, Japan \\ Correspondence should be addressed to Satoru Iizuka, iizuka@ecei.tohoku.ac.jp
}

Received 30 June 2011; Revised 25 October 2011; Accepted 28 October 2011

Academic Editor: Takuya Tsuzuki

Copyright ( $\odot 2012$ S. Iizuka and T. Muraoka. This is an open access article distributed under the Creative Commons Attribution License, which permits unrestricted use, distribution, and reproduction in any medium, provided the original work is properly cited.

Spherical MgO nanoparticles with a hollow inside, that is, $\mathrm{MgO}$ hollow nanospheres, were created in $\mathrm{Ar} / \mathrm{O}_{2}$ plasma produced by radio frequency (RF) impulse discharge using a $\mathrm{Mg}$ rod electrode. The hollow nanospheres were found on the $\mathrm{SiO}_{2}$ plates placed near the powered $\mathrm{Mg}$ electrode. The electron refraction pattern showed that each nanosphere was made of a single crystal of $\mathrm{MgO}$. Since the shape was spherical, these nanoparticles seemed to be created during the levitation in the plasma without touching any walls. The formation mechanism with a quasiliquid cooling model was also discussed.

\section{Introduction}

Magnesium oxide $(\mathrm{MgO})$ has been utilized as a transparent film with favourable secondary electron emission coefficient for a flat plasma display panel [1]. $\mathrm{MgO}$ is also used as a buffer layer for the deposition of high $\mathrm{T}_{\mathrm{c}}$ superconducting films and perovskite-type ferroelectric films. $\mathrm{MgO}$ has been widely used as a refractory material in steel manufacture because of its high corrosion resistance and high-melting point. $\mathrm{MgO}$ is also used as an optical transmitter and as a substrate for thin film growth. Various methods for the production of $\mathrm{MgO}$ films were reported [2-5], where various morphologies of $\mathrm{MgO}$ films, such as flat thin films, whiskers, fishbone fractal nanostructures, and nanofibers, were observed. Photoluminesence emission spectra in the UV range were dependent on the size of $\mathrm{MgO}$ nanoparticles [6].

Here, a special shape of $\mathrm{MgO}$ nanoparticles with a hollow space inside, that is, a hollow nanosphere or a spherical nanoshell, is reported. Moreover, each particle is made of a single crystal of $\mathrm{MgO}$ in spite of its spherical shape. This kind of structure can be used to form an optical scattering surface when they are coated on the surface homogeneously. The mass to volume ratio can be reduced compared to a conventional packed sphere. An improvement of electronic properties such as field electron emission efficiency might be expected. Another possible application includes packing of foreign materials in the hollow.

Concerning with the formation of hollow microspheres, several works have been reported. Ferrite hollow spheres were prepared and fabricated by coating ferrite nanoparticles on the surface of polystyrene spheres and removing of the core polymer subsequently [7]. $\mathrm{SiO}_{2}$ hollow spheres were also prepared by heat treatment of a mixture composed of $\mathrm{SiCl}_{4}$ and carbon microsphears [8]. Double shell hollow spheres were prepared by encapsulating the polymeric hollow spheres with a $\mathrm{TiO}_{2}$ shell [9]. By using a pulsed laser deposition method, hollow $\mathrm{ZnO}$ spheres have been prepared after annealing the depositions [10]. All of these experiments employed an additional chemical and heat treatment. Recently, one-step synthesis of $\mathrm{MgO}$ hollow nanospheres were demonstrated by a pulsed-laser ablation method, where $\mathrm{Mg}$ target was melted, followed by surface oxidization [11]. However, only a simple sputtering method was employed in $\mathrm{Ar} / \mathrm{O}_{2}$ plasma without chemical and thermal processes. The formation technique was quite different from the above methods. 

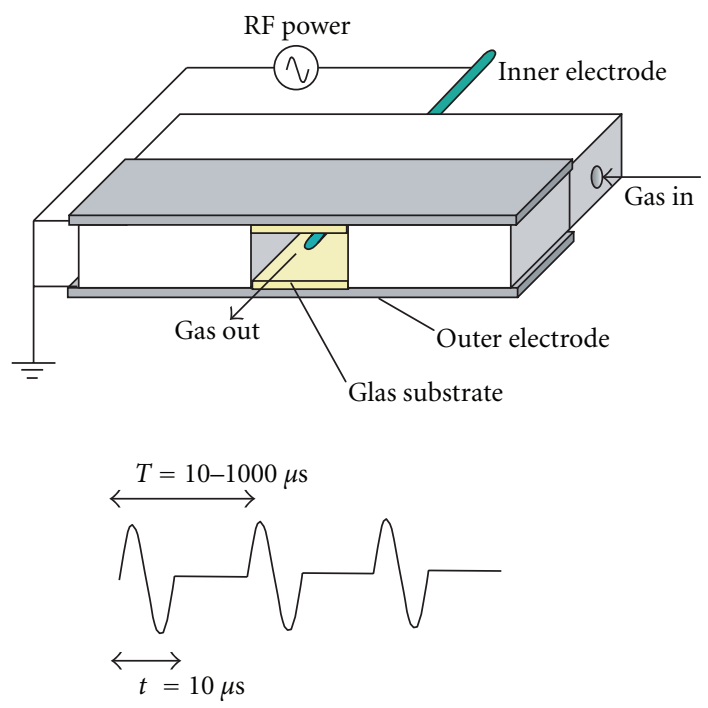

Figure 1: RF impulse discharge system with plate and rod electrodes. The gas was fed between glass plates with narrow gap where the electric impulse discharge takes place. Inset shows a waveform of the applied voltage.

\section{Experimental Setup}

The experimental configuration is shown in Figure 1 [12]. Using this system, deposition materials on the flat glass plates made of $\mathrm{SiO}_{2}$ can be easily analysed. Two glass plates were placed with a narrow spacing gap as shown in Figure 1. The spacing between the glass plates was fixed at $2 \mathrm{~mm}$. To ignite a discharge, a powered rod electrode was introduced between the two glass plates. Another two grounded stainless plate electrodes that sandwich the glass plates were also placed. The inner rod electrode was made of a $\mathrm{Mg}$ rod with a diameter of $1.7 \mathrm{~mm}$. The outer stainless electrode was not fixed, so that the distance between the inner and outer electrodes could be varied in the axial direction. In this experiment, however, the distance between the inner and outer electrodes was fixed at $5 \mathrm{~mm}$.

The entire electrode system shown in Figure 1 was set inside a cylindrical vacuum chamber of $50 \mathrm{~cm}$ in diameter and $20 \mathrm{~cm}$ in height. The working gases of $\mathrm{Ar}$ and $\mathrm{O}_{2}$ were introduced into a mixing vessel through mass flow controllers independently, and finally the mixed gas was fed to the discharge region through a gas inlet tube connected to the inner electrode, as shown in Figure 1. The outgoing gas from the discharge region was directly drained into the chamber and evacuated by a rotary pump. Since the length of the glass plate was short $(\approx 10 \mathrm{~mm})$, the pressure in the discharge region was nearly the same as that in the vacuum chamber. The pressure of the chamber was fixed at 0.1 Torr with a total gas flow rate of $20 \mathrm{sccm}$.

RF impulse voltage was directly supplied to the inner electrode through a coaxial cable without using a matching circuit and a blocking condenser, while the outer electrode is grounded. As shown in an inset of Figure 1, the RF impulse power supply provided one cycle of a sinusoidal waveform of
$10 \mu \mathrm{s}$ in width with the repetition frequency $\omega_{R}$. The pulse amplitude and the repetition frequency can be changed. In this experiment, the pulse repetition frequency was fixed at $4.5 \mathrm{kHz}$. The applied voltage can be increased up to $20 \mathrm{kV}$.

The surface morphology was analysed by scanning electron microscopy (SEM) with a resolution of $5.0 \mathrm{~nm}$ and a maximum magnification of 300,000 (JCM-5700, JEOL). The transmission electron microscopy (TEM) analysis was also employed to analyse the crystal structure.

\section{Experimental Results}

Figure 2 shows SEM images of the depositions on the glass plate surface. Many small spherical particles were formed as shown in Figure 2(a). Typical size of the particles was 200-400 nm. Here, spherical nanoparticles with diameters less than $100 \mathrm{~nm}$ could be also observed. Figure 2(b) shows a SEM image in the direction perpendicular to the glass plate surface. The cross-sectional view of these particles indicates that these particles seem to be just put on the glass plate surface. So, it was supposed that these particles were formed during levitation in the plasma discharge, and fallen down and attached to the surface of the glass plate. It was also found that these nanospheres had a quite symmetrical ball structure, and so there was no indication that they were grown up on the surface of the substrate. Therefore, these particles should have grown in the plasma without touching any places. The dependence of the depositions on the experimental parameters was described in [13], together with [12].

In order to evaluate the structure of these spherical particles more in detail, a TEM image is shown in Figure 3, where several spherical nanoparticles can be observed. Note that most of particles are hollow spheres, although the size is not the same; that is, most of these nanoparticles are spherical and include spherical hollow inside. But, some of them have nonuniform shell thickness. In order to check the crystallite of those hollow spheres more in detail, a few particles were chosen as shown in Figure 4(a). The electron refraction pattern for these particles is shown in Figure 4(b), where narrow refraction spots can be clearly observed. In general, the relation $D=\lambda L / r(\mathrm{hkl})$ holds for the electron refraction, where $D$ is radius of the refraction ring, $L$ is the distance between the sample and screen, $\lambda$ is de Brolie wavelength of the electron beam, and $r(\mathrm{hkl})$ is lattice position in the $x, y$, and $z$ directions. It was obtained that $r(111)=$ $0.244 \mathrm{~nm}, r(200)=0.211 \mathrm{~nm}, r(220)=0.149 \mathrm{~nm}, r(311)=$ $0.127 \mathrm{~nm}, r(222)=0.120 \mathrm{~nm}$, and $r(400)=0.105 \mathrm{~nm}$. The positions of these spots are found to be exactly coincident with the radii of the rings related with the $\mathrm{MgO}$ with lattice constant $a=0.4203 \mathrm{~nm}$. Therefore, it is concluded that each hollow spherical particle is made of a single crystal of $\mathrm{MgO}$.

Figure 5 shows the relation between outer diameter $D$ and inner hollow diameter $d$ of $\mathrm{MgO}$ nanospheres. The hollow diameter $d$ increases almost in proportion with an increase of the outer diameter $D$. From a straight line drawn in the figure, the ratio $d / D$ was found to be about 0.6 , and the ratio $d / D$ was almost independent of the particle size; 


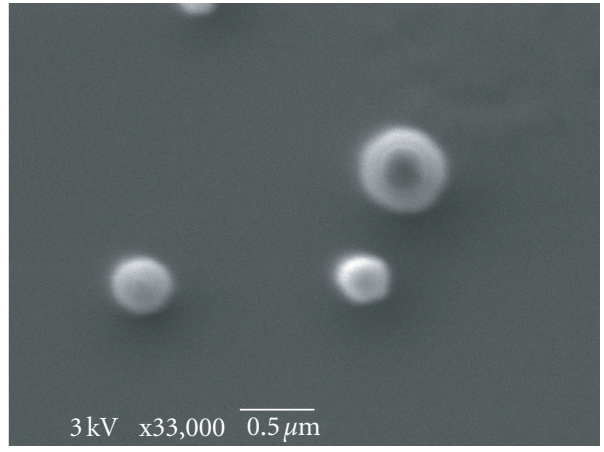

(a)

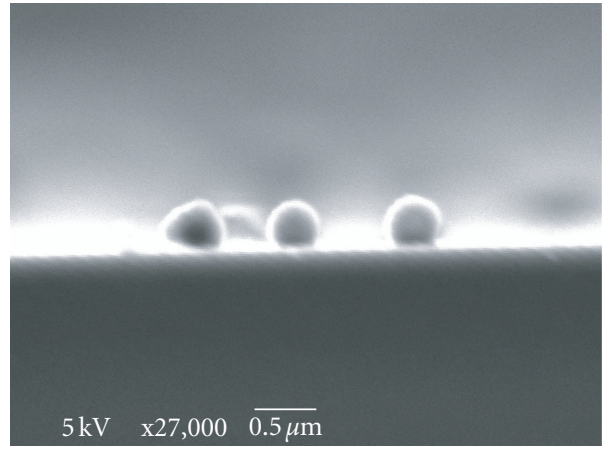

(b)

FIgURE 2: SEM images of the nanoparticles deposited when $\mathrm{Ar} / \mathrm{O}_{2}$ mixing ratio is $3 / 1$ and total pressure is 0.1 Torr. (a) top view, (b) side view. The particles are spherical shape.

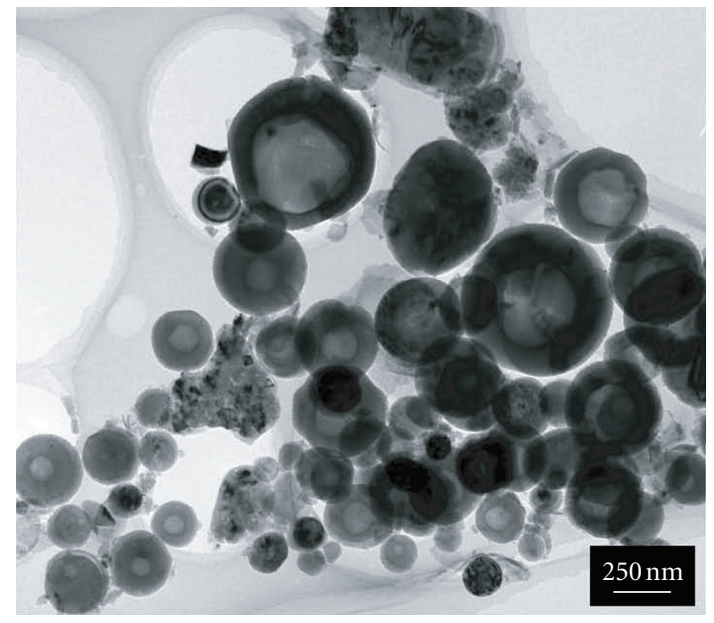

FIGURE 3: TEM images of nanoparticles deposited near the electrode when $\mathrm{Ar} / \mathrm{O}_{2}$ mixing ratio is $3 / 1$ and total pressure is 0.1 Torr. The size of nanoparticles is distributed mostly in the range $50-500 \mathrm{~nm}$. Most of particles have spherical hollow inside.

that is, larger particles had a larger hollow inside. However, most of the particles smaller than $50 \mathrm{~nm}$ seemed to have no detectable hollow inside. These phenomena would be closely related with the formation mechanism of the hollow nanospheres, as discussed in the next section.

The SEM image in the region of thick deposition is shown in Figure 6. It can be confirmed that the depositions are in principle consisted of many small nanoparticles. Moreover, a few particles in Figure 6 are found to have an open hole on the surface. This indicates that a thin part of the shell layer surrounding the particle has been broken by chance, and the inner hollow space was exposed through an open hole. This structure also confirms that these particles are basically hollow particles.

\section{Discussion}

Here, the formation mechanism of these hollow $\mathrm{MgO}$ nanospheres is discussed. A diffusion model of oxygen into a melted $\mathrm{Mg}$ nanosphere was proposed for a formation of $\mathrm{MgO}$ hollow nanosphere in pulsed-laser deposition [11]. During the cooling, oxygen in gas phase penetrated into $\mathrm{Mg}$ nanospheres to form a MgO layer on the surface. On the contrary, $\mathrm{Mg}$ atoms in the core region diffused toward the surface region, leaving a void in the core. This model could not be directly applied to the present study, because melted metallic Mg nanoparticles were not created initially. Instead, nanoparticles contain both $\mathrm{Mg}$ and $\mathrm{O}$ atoms from the beginning, because the reactions between $\mathrm{Mg}$ and $\mathrm{O}$ would occur immediately after $\mathrm{Mg}$ sputtering from the $\mathrm{Mg}$ rod electrode in the discharge plasma.

In this study, the following model was considered for a formation of hollow $\mathrm{MgO}$ nanospheres. Owing to the sheath potential in front of the $\mathrm{Mg}$ rod electrode during the discharge, $\mathrm{Mg}$ atoms are sputtered by energetic Ar ions. These $\mathrm{Mg}$ atoms react with $\mathrm{O}$ atoms in the plasma to form $\mathrm{MgO}$ nuclei. Such $\mathrm{MgO}$ nanoclusters would coagulate and grow in the plasma, and then nanoparticles containing $\mathrm{Mg}$ and $\mathrm{O}$ atoms were produced. Note that in the plasma, strong local electric filed occurs in front of the levitated nanoparticles due to a sheath formation. Therefore, Ar ions are accelerated towards the surface of nanoparticle and transfer kinetic energy to nanoparticles, then the nanoparticles will be heated up as discussed below and will reach a quasiliquid state. It was also noted that these nanoparticles were charged up negatively by an effect of electrons in the discharge. Therefore, they can be confined electrically within positive plasma potential for a long period. Since the nanoparticles are in quasiliquid state, the shape will be spherical, as schematically illustrated in Figure 7(a).

Since the impulse discharge was employed, these nanospheres would have a chance to escape from the discharge region during discharge off-interval. In this case, these particles will be in turn cooled down owing to the collisions with neutral Ar atoms before arriving at the surface of the glass plate. The solidification of nanoparticles will start from the particle surface, as shown in Figure 7(b). During this process, $\mathrm{Mg}$ atoms will be subtracted toward the surface for $\mathrm{MgO}$ crystal formation, because the number of $\mathrm{Mg}$ atoms would be lacked near the surface, compared 


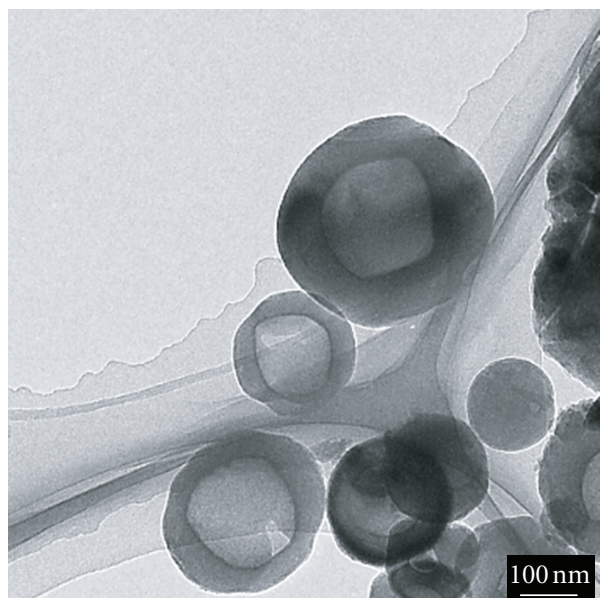

(a)

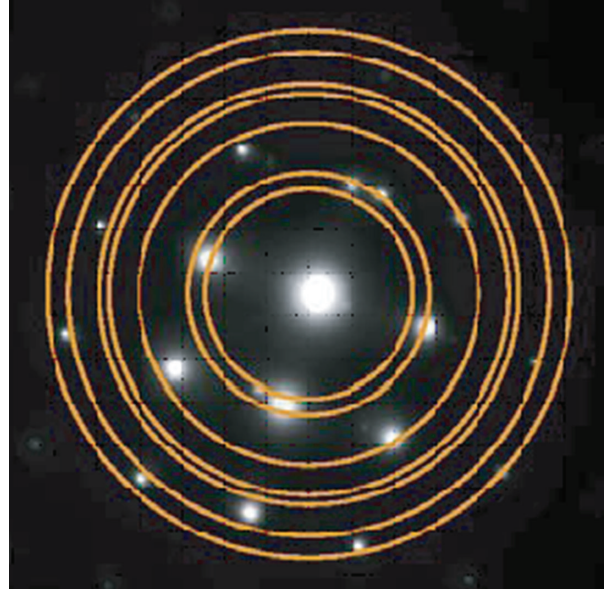

(b)

FIgURE 4: (a) TEM image of a few nanoparticles and (b) electron refraction pattern for these particles. The rings radii correspond to refraction positions for $\mathrm{MgO}$ crystal. This dotted pattern shows that the spherical hollow nanoparticles are consisted of single crystal of $\mathrm{MgO}$.

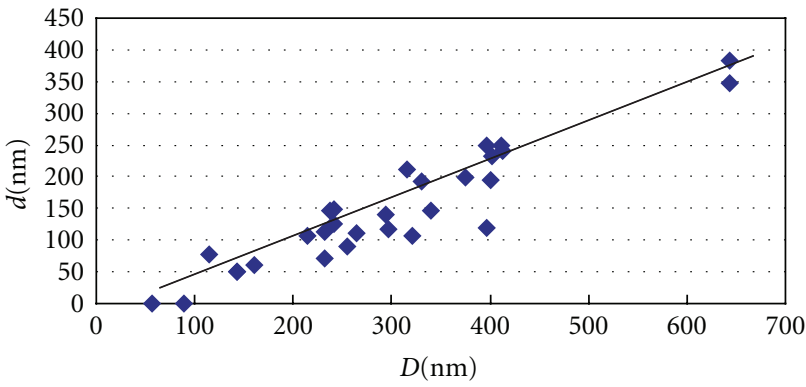

Figure 5: Relation between the diameters $D$ and $d$ of nanospheres and spherical hollow inside, respectively, for nanospheres deposited under the same condition in Figure 3. Solid line shows a slope of $d / D=0.6$

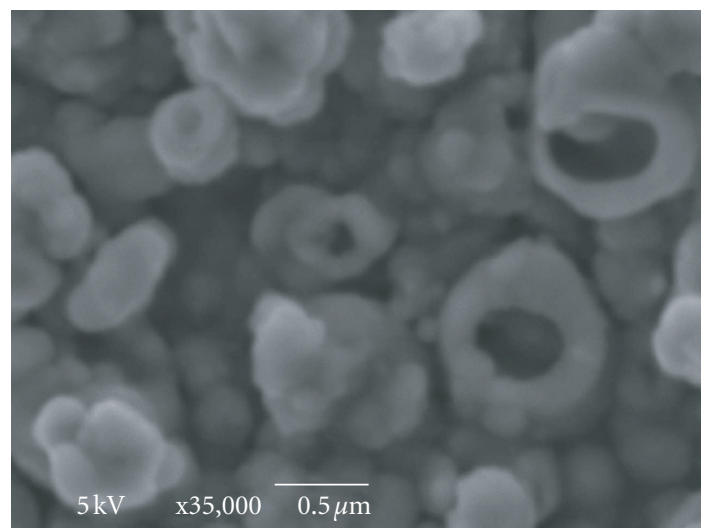

FIGURE 6: SEM images of nanoparticles deposited near the electrode when $\mathrm{Ar} / \mathrm{O}_{2}$ mixing ratio is $3 / 1$ and total pressure is 0.1 Torr. There observed several nanoparticles with circular hole on the surface. These nanoparticles seem to be originally formed as hollow spheres.

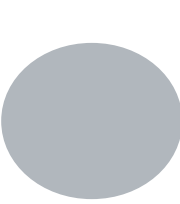

(a)

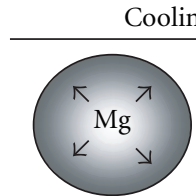

(b)

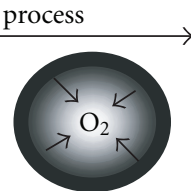

(c)

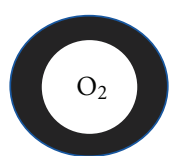

(d)
FIgURE 7: Cooling process of nanoparticles. Liquid, solid, and gas regions are drawn by grey, black, and white, respectively. (a) Liquid $\mathrm{MgO}$ droplets with spherical shape. (b) Outer surface starts being cooled down to form $\mathrm{MgO}$ crystal by collecting $\mathrm{Mg}$ atoms from the central core. (c) The crystallization proceeds toward the core by expelling excess oxygen toward the centre. (d) Hollow nanosphere of single crystal $\mathrm{MgO}$.

with that of oxygen supplied from the gas phase. On the other hand, excess oxygen inside the nanoparticles will be expelled toward the melted core region, because outer surface was solidified and crystallized, as shown in Figure 7(c). This process will lead to a hollow inside filled with oxygen, as shown in Figure 7(d). Since the cooling process is rather slow, compared with the heating process, single crystal structure of $\mathrm{MgO}$ would be formed. Here, it is also noted that in the quasiliquid state, $\mathrm{Mg}$ and $\mathrm{O}$ will be ionised to form $\mathrm{Mg}^{2+}$ and $\mathrm{O}^{2-}$, respectively. The electric force among these ions inside the nanoparticles may also play a key role for driving a collection of $\mathrm{Mg}^{+}$toward the surface (see Figure 7(b)) and an expulsion of excess $\mathrm{O}^{-}$toward the core (see Figure 7(c)) during crystal formation mentioned above.

The energy $W_{i}(\mathrm{~J} / \mathrm{s})$ transferred to the $\mathrm{MgO}$ nanoparticle of radius $R$ per second can be estimated by ion bombardment 
energy $E_{i}=e V_{s}$, and ion flux $J_{i}=n_{i} v_{i}$, that is, $W_{i}=$ $S_{p} E_{i} J_{i}$, where $S_{p}$ is the particle surface area $\left(=4 \pi R^{2}\right), n_{i}$ is ion density, $v_{i}=\sqrt{2 e V_{s} / m_{i}}$ is ion velocity, and $V_{s}$ is ion acceleration voltage in the sheath in front of the nanoparticle. On the other hand, the heat capacity $H\left(\mathrm{~J} /{ }^{\circ} \mathrm{C}\right)$ for increasing the particle temperature is expressed by $H=k M$, where $k\left(\mathrm{~J} / \mathrm{kg}^{\circ} \mathrm{C}\right)$ is specific heat, $M=(4 / 3) \rho_{0} \pi R^{3}$ is mass, and $\rho_{0}$ is specific gravity of $\mathrm{MgO}$. Of course, the other heating mechanisms, such as radiation heating and electron heating and also the other cooling mechanism such as the collisions with neutral $\mathrm{Ar} / \mathrm{O}_{2}$ gas should be also taken into account. Here, however, as a dominant mechanism, the effect of ion bombardment has to be discussed. In the case of a particle of $R=3 \mathrm{~nm}$, for example, it is obtained that $\Delta T / \tau=5.7 \times 10^{2}$ for the time $\tau$ required for increasing the particle temperature $\Delta T$. This shows that an expose of particles to the plasma for $\tau=3.6 \mathrm{~s}$ will lead to an increase of particle temperature by about $\Delta T=1000^{\circ} \mathrm{C}$. Here, the impulse discharge with repetition frequency $\omega_{R}=4.5 \mathrm{kHz}, V_{s}=10 \mathrm{~V}$, and $n_{i}=$ $5 \times 10^{9} / \mathrm{cm}^{3}$ was taken into account. The temperature rises more quickly for smaller particles $(T / \tau \propto 1 / R)$. If the other heating mechanism mentioned above was considered, the temperature rise time might be much shorter. In a typical case, the discharge was continued for 10 minutes. Therefore, nanoparticles growing in the plasma would be heated up by the ion bombardment to reach a quasiliquid state.

The volume of such a quasiliquid spherical droplet of $\mathrm{MgO}$ shown in Figure 7(a) expands as a result of heating. During the cooling, solidification proceeds from the surface to the core region. Therefore, the outer diameter $D$ of particles will be maintained, and only the particles in the core region will be rearranged to form a shell structure of $\mathrm{MgO}$ crystal, as shown in Figure $7(\mathrm{~d})$. If the number of $\mathrm{Mg}$ atoms is preserved during the cooling, a relation $n V=n_{0} V_{0}$ will be hold, where $n$ is $\mathrm{Mg}$ density in the droplet, $V\left(=(4 / 3) \pi(D / 2)^{3}\right)$ is the droplet volume, $n_{0}$ is $\mathrm{Mg}$ density in $\mathrm{MgO}$ crystal, and $V_{0}\left(=(4 / 3) \pi\left[(D / 2)^{3}-(d / 2)^{3}\right]\right)$ is the shell volume. Then, the relation $n / n_{0}=V_{0} / V=$ $1-(d / D)^{3} \approx 0.78$ can be obtained. Here, $d / D \approx 0.6$ is used from Figure 5. Therefore, the number ratio of atoms in the quasiliquid $\mathrm{MgO}$ droplet can be estimated to be $\mathrm{Mg}: \mathrm{O} \approx 1: 1.28$, containing excess oxygen. The density of $\mathrm{Mg}$ in the quasiliquid $\mathrm{MgO}$ droplet would be almost constant, independent of the particle size, as shown in Figure 5. This is consistent with the constancy of the ratio $d / D$.

A following trapping mechanism was considered for the nanoparticles levitated in the plasma. The forces acting on the charged nanoparticles levitated in plasmas were electrostatic force, ion drag force, thermophoresis, gravity, and neutral drag by the gas flow. Here, thermophoresis was negligible because of no heating. Since the nanoparticles were deposited on upper and lower sides of the glass plates placed parallel to the horizontal direction, the gravity was unimportant. Neutral drag by gas injection was also negligible because it acted mainly in the horizontal direction. Therefore, ion drag force would play a key role for the particle transport toward the glass plates.

\section{Conclusion}

In conclusion, RF impulse plasma was produced within a space between narrow glass plates using an $\mathrm{Mg}$ rod electrode system. It was found that hollow nanospheres were deposited on the glass plate surface. The particles were predominantly deposited on the glass substrate close to the inner $\mathrm{Mg}$ rod electrode. From the TEM image and electron refraction patterns, these hollow nanospheres were found to be composed with $\mathrm{MgO}$ single crystal. The average size of the particles was several $100 \mathrm{~nm}$. Further, the formation processes of such spherical particles were discussed. A melting process of the particles by the ion bombardment was considered during the growth of $\mathrm{MgO}$ nuclei and cluster. Cooling process starting from the outer surface of the particles will confine excess oxygen in the core, resulting in formation of a hollow sphere made of $\mathrm{MgO}$ single crystal. It is demonstrated that the RF impulse discharge system employed here is very useful for the formation of $\mathrm{MgO}$ hollow nanospheres.

\section{Acknowledgment}

This work was partially supported by a Grant-in-Aid for Scientific Research from the Ministry of Education, Culture, Sports, Science, and Technology, Japan.

\section{References}

[1] T. Urade, T. Iemori, M. Osawa, N. Nakayama, and I. Morita, "Protecting layer for the dielectric in ac plasma panels," IEEE Transactions on Electron Devices, vol. 23, no. 3, pp. 313-318, 1976.

[2] S. Valeri, S. Altieri, A. Di Bona, C. Giovanardi, and T. S. Moia, "Structural study of thin $\mathrm{MgO}$ layers on $\mathrm{Ag}(001)$ prepared by either MBE or sputter deposition," Thin Solid Films, vol. 400, no. 1-2, pp. 16-21, 2001.

[3] S. A. Chambers, Y. Gao, and Y. Liang, "The early stages of MgO epitaxy on lattice-matched Cr0.7Mo0.3(001)," Surface Science, vol. 339, no. 3, pp. 297-309, 1995.

[4] P. Ghekiere, S. Mahieu, G. de Winter, R. de Gryse, and D. Depla, "Scanning electron microscopy study of the growth mechanism of biaxially aligned magnesium oxide layers grown by unbalanced magnetron sputtering," Thin Solid Films, vol. 493, no. 1-2, pp. 129-134, 2005.

[5] K. P. Kalyanikutty, F. L. Deepak, C. Edem, A. Govindaraj, and C. N. R. Rao, "Carbon-assisted synthesis of nanowires and related nanostructures of MgO," Materials Research Bulletin, vol. 40, no. 5, pp. 831-839, 2005.

[6] S. Stankic, M. Müller, O. Diwald, M. Sterrer, E. Knözinger, and J. Bernardi, "Size-dependent optical properties of $\mathrm{MgO}$ nanocubes," Angewandte Chemie International Edition, vol. 44, no. 31, pp. 4917-4920, 2005.

[7] Y. Zhang, Z. Huang, F. Tang, and J. Ren, "Ferrite hollow spheres with tunable magnetic properties," Thin Solid Films, vol. 515, no. 4, pp. 2555-2561, 2006.

[8] Y. Tan, C. Li, Y. Wang, J. Tang, and X. Ouyang, "Fastresponse and high sensitivity gas sensors based on $\mathrm{SnO}_{2}$ hollow spheres," Thin Solid Films, vol. 516, no. 21, pp. 7840$7843,2008$.

[9] C. Chang, M. Tsai, G. Chen, M. Wu, and T. Hung, "Preparation and properties of porous polyimide films with 
$\mathrm{TiO}_{2}$ /polymer double shell hollow spheres," Thin Solid Films, vol. 517, no. 17, pp. 4966-4969, 2009.

[10] J. Moon, J. Park, S. Lee, and T. Zyung, "Colloidal templating for producing hollow $\mathrm{ZnO}$ shells: fabrication, growth and electrical properties," Thin Solid Films, vol. 517, no. 14, pp. 3904-3907, 2009.

[11] K.-Y. Niu, J. Yang, J. Sun, and X.-W. Du, “One-step synthesis of $\mathrm{MgO}$ hollow nanospheres with blue emission," Nanotechnology, vol. 21, no. 29, article 295604, 2010.

[12] T. Muraoka and S. Iizuka, "MgO microparticle deposition by radio frequency impulse discharge in a small-diameter tube," Japanese Journal of Applied Physics, vol. 48, no. 2, Article ID 025501, 4 pages, 2009.

[13] T. Muraoka, T. Kashimura, and S. Iizuka, "Spherical MgO microparticle deposition by RF impulse discharge with small coaxial electrodes," Thin Solid Films, vol. 518, no. 3, pp. 1012 1015, 2009. 

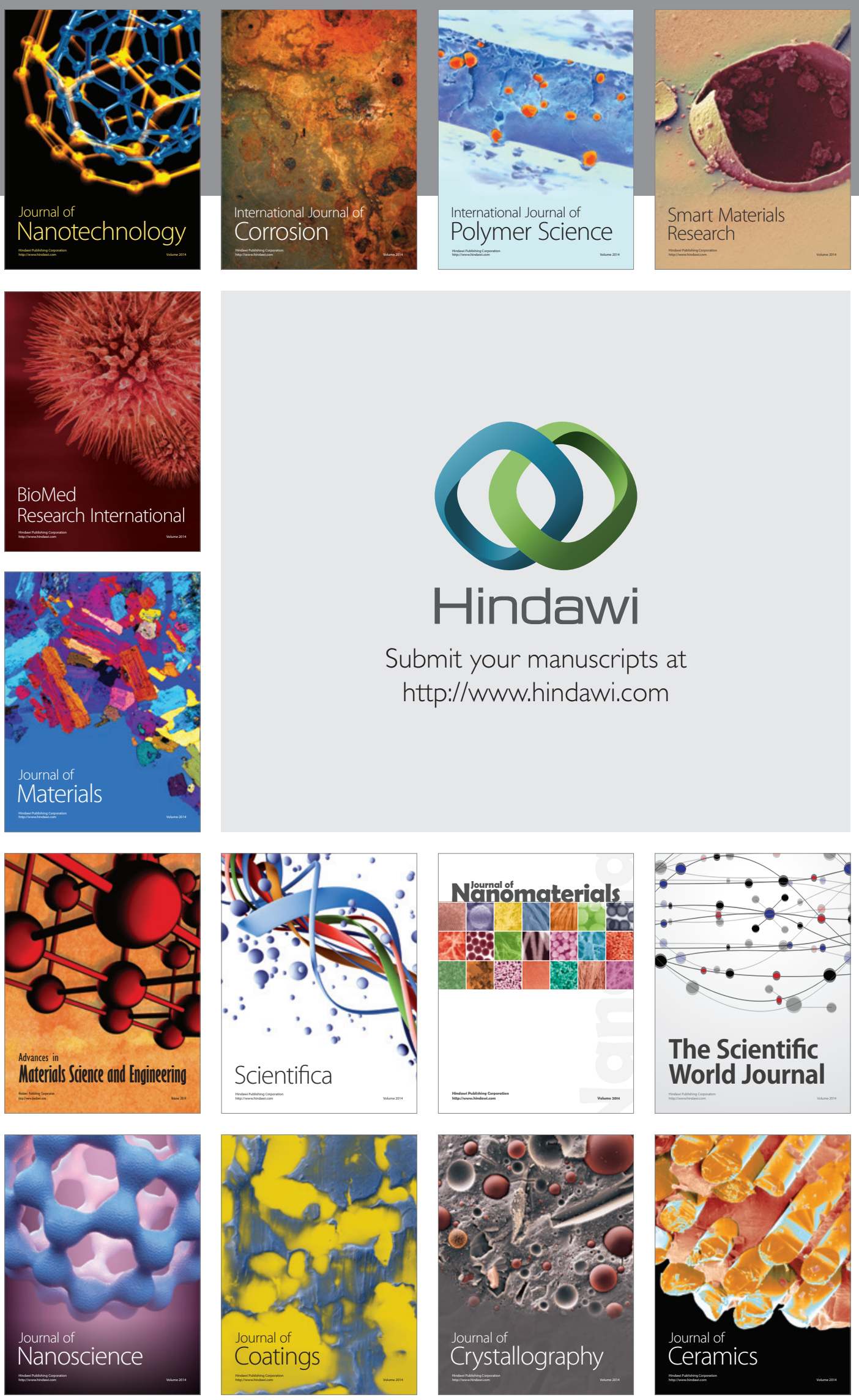

The Scientific World Journal

Submit your manuscripts at

http://www.hindawi.com

\section{World Journal}

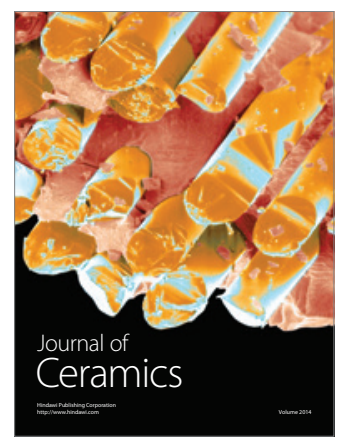

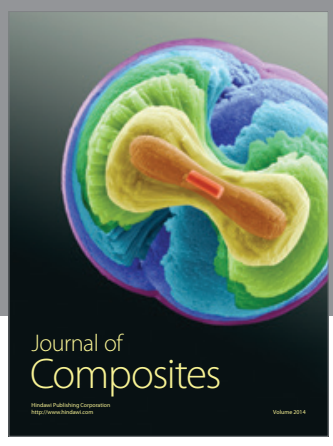
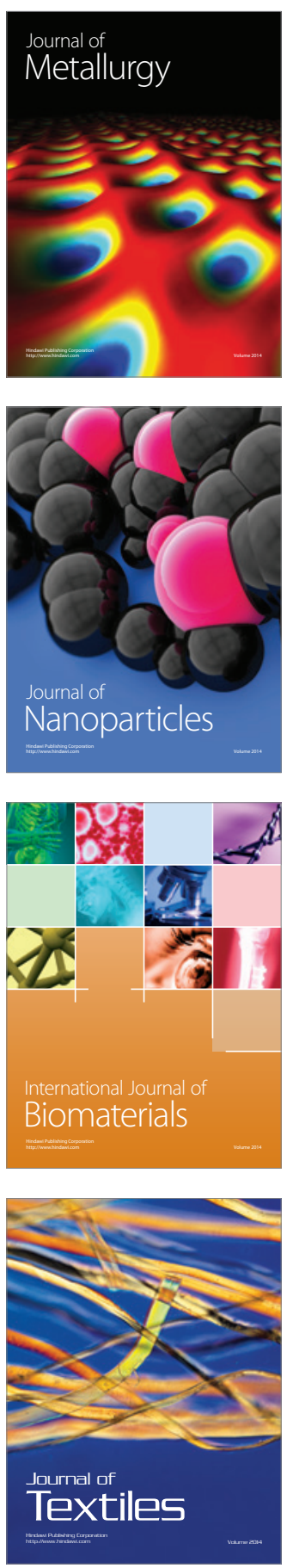